\title{
From the atomic limit to a metal-insulator transition in the Hubbard model
}

\author{
L. Craco and M. A. Gusmão \\ Instituto de Física, Universidade Federal do Rio Grande do Sul, 91501-970 Porto Alegre, Rio Grande do Sul, Brazil
}

(Received 15 December 1994; revised manuscript received 16 March 1995)

\begin{abstract}
Starting from the exact solution of the Hubbard model in the atomic limit, and treating the hopping term as a perturbation by means of a diagram technique, we discuss the electronic structure of the model in some simple approximations. We show that a metal-insulator transition is obtained above a critical value of the on-site Coulomb interaction in a self-consistent evaluation of the oneparticle Green's function to one-loop order. A relationship with results in infinite dimensions is briefly discussed.
\end{abstract}

\section{INTRODUCTION}

Since the early works of Hubbard, ${ }^{1-4}$ much effort has been invested in developing a useful method to study the Hubbard Hamiltonian and related models using perturbation theory around the solution of the atomic limit. ${ }^{1-6}$ The advantage of such an approach lies on the nonperturbative treatment of the on-site Coulomb interaction $U$, which is a desirable feature in the case of strongly correlated systems. However, the price to pay for the inclusion of interaction in the unperturbed Hamiltonian is the breakdown of Wick's theorem, preventing utilization of well-known many-body techniques. Besides that, the simplest approximations tend to retain a strong memory of the atomic limit, favoring an insulating state in the case of a half-filled band. Thus, the search for a metalinsulator transition and Fermi-liquid behavior is not a trivial task.

We will mainly refer here to a systematization of the perturbative treatment of the hopping term presented by Metzner, ${ }^{5}$ whose diagrammatic representation we will adopt to some extent. We will discuss the formal summation of the perturbation series, and develop the simplest approximations, namely, the zero-loop (ZLA) and selfconsistent one-loop (SC1LA) approximations. The first one reproduces the well-known Hubbard I decoupling of the equations of motion, ${ }^{1}$ which predicts an insulating state at half filling for any nonzero value of $U$. The last one shows evidence of a metal-insulator transition for finite $U$, with a metallic state that is not a normal Fermi liquid.

Our analysis will be based on the calculation of finite temperature (Matsubara) Green's functions, from which the retarded Green's functions can be obtained by analytical continuation in frequency space. The insulating or metallic nature of the state of the system will be inferred from the shape of the one-particle density of states (DOS) and frequency dependence of the self-energy.

We will focus on the case of a half-filled band system, and impose a paramagnetic solution. It is well known that this state is unstable against antiferromagnetism in a bipartite tight-binding lattice as considered here, due to the nesting properties of the Fermi surface. Nevertheless, one can assume that a small next-nearest-neighbor hopping will break the nesting condition and stabilize the paramagnetic solution. It is this situation that we have in mind here, and we will be able to compare our results with other approaches where the same kind of assumption is implied. ${ }^{3,7,8}$

The paper is organized as follows. In Sec. II we describe the perturbation method in a general way. The simplest approximations are described in Sec. IV, where a detailed evaluation of the one-loop diagrams is presented. Calculations for the half-filled paramagnetic case are presented in Sec. V. Final comments and a discussion of our results in comparison with other approaches appear in Sec. VI.

\section{PERTURBATION METHOD}

We start with the usual form of the Hubbard Hamiltonian,

$$
\begin{aligned}
H=(\epsilon & -\mu) \sum_{i \sigma} n_{i \sigma}+U \sum_{i} n_{i \uparrow} n_{i \downarrow} \\
& -h \sum_{i}\left(n_{i \uparrow}-n_{i \downarrow}\right)-t \sum_{\langle i j\rangle \sigma} c_{i \sigma}^{\dagger} c_{j \sigma}
\end{aligned}
$$

in standard notation, with explicit inclusion of the chemical potential $\mu$, and with an applied uniform magnetic field $h$, whose role will be discussed later. The first three terms on the right-hand side of Eq. (1) will be considered as the unperturbed Hamiltonian, while the last one will be the perturbation.

The temperature-dependent one-particle Green's function (1PGF) is defined as

$$
G_{i j \sigma}(\tau)=-\left\langle\hat{T} c_{i \sigma}(\tau) c_{j \sigma}^{\dagger}(0)\right\rangle,
$$

where $\hat{T}$ stands for the time ordering operator for fermions, and the "time" $\tau$ is defined in the interval $[-\beta, \beta], \beta$ being the inverse temperature. Applying the 
usual perturbation theory for many-body systems, ${ }^{9}$ we can rewrite Eq. (2) in the form

$$
G_{i j \sigma}(\tau)=-\frac{\left\langle\hat{T} c_{i \sigma}(\tau) c_{j \sigma}^{\dagger}(0) S(\beta)\right\rangle_{0}}{\langle S(\beta)\rangle_{0}}
$$

where the subscript zero indicates that the average is performed with respect to the unperturbed Hamiltonian, which also defines the time dependence of the operators. Representing by $H_{1}$ the hopping term in Eq. $(1), S(\beta)$ is given by

$$
S(\beta)=\hat{T} \exp \left\{-\int_{0}^{\beta} d \tau^{\prime} H_{1}\left(\tau^{\prime}\right)\right\},
$$

and expanded in powers of $H_{1}$.

The perturbation series for the 1PGF can be expressed in terms of local (unperturbed) cumulant averages, and represented by means of Feynman-like diagrams, as discussed in detail by Metzner. ${ }^{5}$ The relevant diagrams for bipartite lattices up to fourth order are shown in Ref. 5. Inspection of the general structure of those diagrams shows that they are made up of irreducible parts connected together by hopping lines. The irreducible parts cannot be divided in two pieces by the operation of cutting a single hopping line. With such a structure, the entire perturbation series can be formally summed by means of a Dyson-like equation. After Fourier transforming in space and time, we can write

$$
G_{\mathbf{k} \sigma}\left(i \omega_{n}\right)=\mathcal{G}_{\mathbf{k} \sigma}\left(i \omega_{n}\right)+\mathcal{G}_{\mathbf{k} \sigma}\left(i \omega_{n}\right) \epsilon_{\mathbf{k}} G_{\mathbf{k} \sigma}\left(i \omega_{n}\right)
$$

where $\omega_{n}$ is a fermionic Matsubara frequency, $\epsilon_{\mathbf{k}}$ is the tight-binding dispersion relation, which gives the allowed energies for noninteracting particles in the lattice, and $\mathcal{G}_{\mathbf{k} \sigma}\left(i \omega_{n}\right)$ stands for the irreducible part of the 1PGF, whose first few diagrams are shown in Fig. 1.

The formal solution of Eq. (5) is

$$
G_{\mathbf{k} \sigma}\left(i \omega_{n}\right)=\frac{1}{\left[\mathcal{G}_{\mathbf{k} \sigma}\left(i \omega_{n}\right)\right]^{-1}-\epsilon_{\mathbf{k}}} .
$$
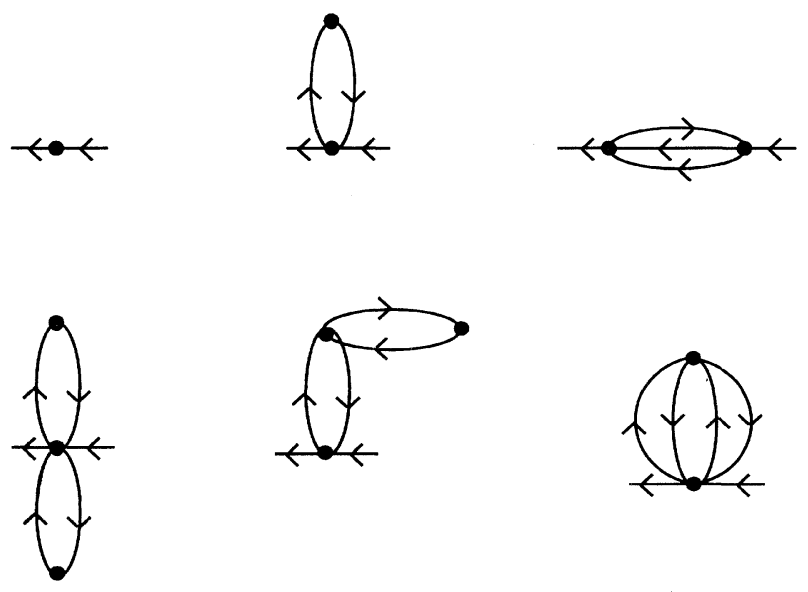

FIG. 1. Diagrams of the irreducible Green's function $\mathcal{G}$ up to fourth order in the hopping.
It is possible to express this 1PGF in the usual way in terms of a self-energy, provided the latter is defined as

$$
\Sigma_{\mathbf{k} \sigma}\left(i \omega_{n}\right) \equiv i \omega_{n}-\left[\mathcal{G}_{\mathbf{k} \sigma}\left(i \omega_{n}\right)\right]^{-1}+\mu .
$$

\section{ONE-PARTICLE DENSITY OF STATES}

We can analytically continue $G_{\mathbf{k} \sigma}\left(i \omega_{n}\right)$ to the entire complex plane, obtaining a function $G_{\mathbf{k} \sigma}(z)$. Then, the retarded Green's function is just $G_{\mathbf{k} \sigma}\left(\omega+i 0^{+}\right)$, for real $\omega$, and the single-particle density of states (for particles of spin $\sigma$ ) is given by

$$
\rho_{\sigma}(\omega)=-\frac{1}{\pi} \frac{1}{N} \sum_{\mathbf{k}} \operatorname{Im} G_{\mathbf{k} \sigma}\left(\omega+i 0^{+}\right) .
$$

This analytic continuation may be highly nontrivial, depending on the kind of approximation employed. For some approximations the simple substitution of $\omega+i \delta$ $(\delta \rightarrow 0)$ for $i \omega_{n}$ in the final expressions works perfectly well. It fails, however, in certain cases, mainly when one utilizes self-consistent solutions, as will be the case in our SC1LA. This failure manifests itself in the breakdown of the sum rule $\int \rho(\omega) d \omega=1$. In principle, we could be in the presence of a nonconserving approximation. ${ }^{10}$ This is not true for our SC1LA, since the number of particles calculated by a direct (numerical) summation of the 1PGF over Matsubara frequencies is consistent with the choice of chemical potential. The identity

$$
\left\langle n_{\sigma}\right\rangle=\frac{1}{N} \sum_{\mathbf{k}} \frac{1}{\beta} \sum_{\omega_{n}} G_{\mathbf{k} \sigma}\left(i \omega_{n}\right)=\int d \omega \rho_{\sigma}(\omega) f(\omega)
$$

has to be satisfied if the spectral representation

$$
G_{\mathbf{k} \sigma}(z) \equiv \int d \omega^{\prime} \frac{A_{\mathbf{k} \sigma}\left(\omega^{\prime}\right)}{z-\omega^{\prime}}
$$

is utilized. Notice that the spectral function $A_{\mathbf{k} \sigma}(\omega)$ is related to the imaginary part of the 1 PGF through

$$
A_{\mathbf{k} \sigma}(\omega)=-\frac{1}{\pi} \operatorname{Im} G_{\mathbf{k} \sigma}\left(\omega+i 0^{+}\right),
$$

and its sum over momentum gives the single-particle density of states [see Eq. (8)].

Given that the first equality in Eq. (9) is correctly satisfied, the failure of the second equality must lie on the evaluation of $A_{\mathbf{k} \sigma}(\omega)$ through Eq. (11), with the implied analytic continuation on the complex frequency plane.

By summing Eq. (10) over momentum, we can directly relate the local Matsubara Green's function with the DOS through

$$
G_{i i \sigma}\left(i \omega_{n}\right)=\int d \omega \frac{\rho_{\sigma}(\omega)}{i \omega_{n}-\omega} .
$$

Our method gives us the values of the left-hand side, while we want to know the function $\rho_{\sigma}(\omega)$ appearing on the right-hand side. We are thus faced with the problem of inverting a Hilbert transform. This is a common problem in image reconstruction, ${ }^{11}$ as well as in the process of 
obtaining densities of states from Monte Carlo data for Matsubara Green's functions. ${ }^{12}$ In both cases one utilizes the so-called maximum entropy method to obtain the desired image [in our case, $\rho_{\sigma}(\omega)$ ] starting from a default model (which we have chosen as the result of the direct substitution $\left.i \omega_{n} \rightarrow \omega+i \delta\right)$. In the next section we will show a practical example in which this method provided good results.

\section{SIMPLE APPROXIMATIONS}

The simplest approximation is obtained when one considers just the first diagram in Fig. 1, using the zerothorder solution as the irreducible part of the 1PGF. This takes into account the sum of all zero-loop diagrams. For this reason we call it zero-loop approximation. Actually, one has to renormalize the average number of particles appearing in the zeroth-order Green's function,

$$
\mathcal{G}_{\sigma}^{0}\left(i \omega_{n}\right)=\frac{1-\left\langle n_{\bar{\sigma}}\right\rangle}{i \omega_{n}-\varepsilon+\mu}+\frac{\left\langle n_{\bar{\sigma}}\right\rangle}{i \omega_{n}-\varepsilon-U+\mu},
$$

calculating it self-consistently. This is necessary because for a given chemical potential the number of particles in the atomic limit and in the lattice can be quite different. When considering corrections to the ZLA, one has to be careful and subtract the parts of new diagrams that account for this renormalization of the number of particles in order to avoid double counting their contribution. This point is discussed in further detail in the Appendix, in connection with the calculation of the one-loop correction.

The ZLA reproduces exactly the results of the so-called Hubbard I decoupling of the equations of motion for the 1PGF. ${ }^{1}$ This approximation gives qualitatively the expected behavior in the strong correlation limit $U \gg t$, where a lower and an upper Hubbard subband are separated by a correlation gap in the single-particle DOS. However, this structure appears for any nonzero value of $U$, with the gap collapsing only strictly at $U=0$, where the exact tight-binding band is recovered. An expected metal-insulator transition at some finite $U$ is not present.

One-loop corrections to the ZLA are obtained by adding to the irreducible Green's function $\mathcal{G}_{\sigma}\left(i \omega_{n}\right)$ the diagram depicted in Fig. 2. In that diagram the double line represents the fully renormalized 1PGF (with important subtractions that will be discussed later), and the problem must be solved self-consistently. In this case one has to evaluate the local cumulant average of four fermion operators

$$
\Gamma_{\sigma \sigma^{\prime}}^{(4)}\left(\tau, \tau_{1}, \tau_{2}\right) \equiv\left\langle\hat{T} c_{\sigma}(\tau) c_{\sigma^{\prime}}^{\dagger}\left(\tau_{1}\right) c_{\sigma^{\prime}}\left(\tau_{2}\right) c_{\sigma}^{\dagger}(0)\right\rangle_{0 c},
$$

where we have dropped the site index. To calculate it we rewrite the fermion operators in terms of Hubbard operators, ${ }^{2}$ and utilize the standard algebra for the latter, as well as a generalized Wick's theorem. ${ }^{13}$ Although straightforward, some details of this calculation are worth making explicit, mainly a nonstandard intrasite diagrammatics, and the important point on subtractions of contributions accounted for by the renormalization of the

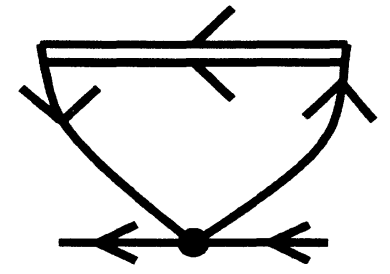

FIG. 2. Fully renormalized one-loop diagram for the irreducible 1PGF. The double line represents a complete Green's function, with subtractions that are discussed in the text.

average number of particles in the zeroth-order Green's function. For this reason, we develop it more or less extensively in the Appendix.

We can write the irreducible Green's function to oneloop order as

$$
\mathcal{G}_{\sigma}^{1 L}\left(i \omega_{n}\right)=\mathcal{G}_{\sigma}^{0}\left(i \omega_{n}\right)+\sum_{\sigma^{\prime}} \Lambda_{\sigma \sigma^{\prime}}\left(i \omega_{n}\right)
$$

where

$$
\Lambda_{\sigma \sigma^{\prime}}\left(i \omega_{n}\right) \equiv \frac{1}{\beta} \sum_{\omega_{l}} \Gamma_{\sigma \sigma^{\prime}}^{(4)}\left(i \omega_{n}, i \omega_{l}\right) H_{\sigma^{\prime}}\left(i \omega_{l}\right)
$$

Here $\Gamma_{\sigma \sigma^{\prime}}^{(4)}\left(i \omega_{n}, i \omega_{l}\right)$ is the Fourier transform of (14), and $H_{\sigma^{\prime}}\left(i \omega_{l}\right)$ stands for the contribution of the loop in Fig. 2. As we mentioned before, the double line that appears in that diagram is to be interpreted as the fully renormalized 1PGF with some important subtractions. To see what these subtractions are we must remember that $G_{\mathbf{k} \sigma}\left(i \omega_{n}\right)$ is the sum of all possible chains of irreducible local Green's functions, $\mathcal{G}_{\sigma}\left(i \omega_{n}\right)$, connected by hopping lines. In order to count only the one-loop contribution in Fig. 2 we must not allow any of the $\mathcal{G}_{\sigma}$ 's of those chains to belong to the site where the average $\Gamma^{4}$ is being evaluated. Notice that this subtraction is necessary only because we are renormalizing the intraloop Green's function. If we counted diagrams order by order the necessary subtractions would be automatically accounted for by the cumulant averages. In fact, we are just rearranging the cumulant expansion to explicitly avoid counting "paths" that come back to the original site. Instead, the normal cumulant expansion would add corrections for their wrong contributions at higher-order terms. The confirmation that this is a better choice comes from the form of the DOS obtained with each procedure, as we will show below. Without any subtractions, $H_{\sigma}\left(i \omega_{n}\right)$ would be replaced by

$$
\tilde{H}_{\sigma}\left(i \omega_{n}\right)=\frac{1}{N} \sum_{\mathbf{k}} \varepsilon_{\mathbf{k}}^{2} G_{\mathbf{k} \sigma}\left(i \omega_{n}\right) .
$$

For each intermediate visit to the reference site in the process of building up $G_{\mathbf{k} \sigma}\left(i \omega_{n}\right)$ we have to subtract $H_{\sigma}\left(i \omega_{n}\right) \mathcal{G}_{\sigma}\left(i \omega_{n}\right) H_{\sigma}\left(i \omega_{n}\right)$. Continuing this process, we can see that

$$
\tilde{H}_{\sigma}\left(i \omega_{n}\right)=H_{\sigma}\left(i \omega_{n}\right)+H_{\sigma}\left(i \omega_{n}\right) \mathcal{G}_{\sigma}\left(i \omega_{n}\right) \tilde{H}_{\sigma}\left(i \omega_{n}\right)
$$


or

$$
H_{\sigma}\left(i \omega_{n}\right)=\frac{\tilde{H}_{\sigma}\left(i \omega_{n}\right)}{1+\tilde{H}_{\sigma}\left(i \omega_{n}\right) \mathcal{G}_{\sigma}\left(i \omega_{n}\right)} .
$$

Utilizing Eq. (6) for the general form of $G_{\mathbf{k} \sigma}\left(i \omega_{n}\right)$ we get

$$
\tilde{H}_{\sigma}\left(i \omega_{n}\right)=\frac{1}{\mathcal{G}_{\sigma}\left(i \omega_{n}\right)}\left[\frac{G_{i i \sigma}\left(i \omega_{n}\right)}{\mathcal{G}_{\sigma}\left(i \omega_{n}\right)}-1\right],
$$

and

$$
H_{\sigma}\left(i \omega_{n}\right)=\frac{1}{\mathcal{G}_{\sigma}\left(i \omega_{n}\right)}-\frac{1}{G_{i i \sigma}\left(i \omega_{n}\right)}
$$

where $G_{i i \sigma}\left(i \omega_{n}\right) \equiv \frac{1}{N} \sum_{\mathbf{k}} G_{\mathbf{k} \sigma}\left(i \omega_{n}\right)$ is the fully renormalized local Green's function.

With $H_{\sigma}\left(i \omega_{n}\right)$ given by Eq. (21), and utilizing $\Gamma_{\sigma \sigma^{\prime}}^{(4)}\left(i \omega_{n}, i \omega_{l}\right)$ as calculated in the Appendix, we obtain

$$
\begin{aligned}
\Lambda_{\sigma \sigma}\left(i \omega_{n}\right)= & \left\langle n_{\bar{\sigma}}\right\rangle\left\langle 1-n_{\bar{\sigma}}\right\rangle\left[g_{\bar{\sigma} 2}\left(i \omega_{n}\right)-g_{0 \sigma}\left(i \omega_{n}\right)\right]^{2} \\
& \times H_{\sigma}\left(i \omega_{n}\right)
\end{aligned}
$$

and

$$
\begin{gathered}
\Lambda_{\sigma \bar{\sigma}}\left(i \omega_{n}\right)=\left[g_{\bar{\sigma} 2}\left(i \omega_{n}\right)-g_{0 \sigma}\left(i \omega_{n}\right)\right] \frac{1}{\beta} \sum_{\omega_{l}}\left\{\left[\mathcal{G}_{\sigma}^{0}\left(i \omega_{n}\right)+\mathcal{G}_{\bar{\sigma}}^{0}\left(i \omega_{l}\right)\right] g_{02}\left(i \omega_{n}+i \omega_{l}\right)\right. \\
\left.+\left[\mathcal{G}_{\sigma}^{0}\left(i \omega_{n}\right)-\mathcal{G}_{\bar{\sigma}}^{0}\left(i \omega_{l}\right)\right] g_{\bar{\sigma} \sigma}\left(i \omega_{n}-i \omega_{l}\right)\right\} H_{\bar{\sigma}}\left(i \omega_{l}\right)
\end{gathered}
$$

which are used in Eq. (15) to give the self-consistent oneloop correction to the irreducible part of the 1PGF.

Equation (23) shows why it was necessary to include an external magnetic field in the problem, since the function $g_{\tilde{\sigma} \sigma}\left(i \omega_{n}-i \omega_{l}\right)$ for $\omega_{l}=\omega_{n}$ diverges in the zero-field limit. Thus, when considering the absence of an applied field, the limit $h \rightarrow 0$ must be taken with some care. Also, the function $g_{02}\left(i \omega_{n}+i \omega_{l}\right)$ diverges for $\omega_{l}=-\omega_{n}$ in the halffilled band case, which makes this another limit that must be approached with care. These divergencies reflect the spin-inversion and particle-hole symmetries of the unperturbed Hamiltonian. At zero magnetic field local singly occupied states are degenerate, so that spin-reversal excitations are massless. Similarly, at half filling both empty or doubly occupied states are degenerate, and local excitations corresponding to transitions between these two states become massless too. The contributions of such (virtual) massless excitations to the renormalization of the single-particle propagator should not introduce new singularities. Thus, solving the problem for an arbitrary band filling and in the presence of an external field constitutes a regularization procedure that yields finite corrections in the limits commented above.

\section{HALF-FILLED BAND, PARAMAGNETIC CASE}

Without loss of generality, we will chose $\varepsilon=-U / 2$, so that we have $\mu=0$ for $n=1$, where $n \equiv \sum_{\sigma}\left\langle n_{\sigma}\right\rangle$ is the total number of particles. There are two delicate limits that one has to take in Eq. (23), which are $\mu \rightarrow 0$ (together with $\left.\left\langle n_{\sigma}\right\rangle \rightarrow 1 / 2\right)$ and $h \rightarrow 0$. One of the problems is the divergence of $g_{\bar{\sigma} \sigma}\left(i \omega_{n}-i \omega_{l}\right)$ in the limit $h \rightarrow 0$ for $\omega_{n}=\omega_{l}$. However, simple algebraic manipulations show that the prefactor $\mathcal{G}_{\sigma}^{0}\left(i \omega_{n}\right)-\mathcal{G}_{\bar{\sigma}}^{0}\left(i \omega_{n}\right)$ is proportional to $\left\langle n_{\sigma}-n_{\bar{\sigma}}\right\rangle$, the local magnetization, which goes to zero lin- early with $h$ in the paramagnetic case. Notice that this would not be the case in an antiferromagnetic solution, and it would be necessary to consider higher-order terms that should renormalize $h$ by the addition of an internal effective field, presumably proportional to the local magnetization. As far as the half-filled limit is concerned, the divergence of $g_{02}\left(i \omega_{n}+i \omega_{l}\right)$ for $\omega_{l}=-\omega_{n}$ when $\mu \rightarrow 0$ is compensated by the fact that $\mathcal{G}_{\sigma}^{0}\left(i \omega_{n}\right)+\mathcal{G}_{\bar{\sigma}}^{0}\left(-i \omega_{n}\right)$ is proportional to $\left\langle 1-n_{\sigma}-n_{\bar{\sigma}}\right\rangle$, which goes to zero with $\mu$. By studying numerically these two limits we observed that the contribution of $\Lambda_{\sigma \bar{\sigma}}\left(i \omega_{n}\right)$ tends to be much smaller than that of $\Lambda_{\sigma \sigma}\left(i \omega_{n}\right)$ for low temperatures. Based on this, and for reasons of simplicity, we will keep only the equal spin term in the remaining of this calculation.

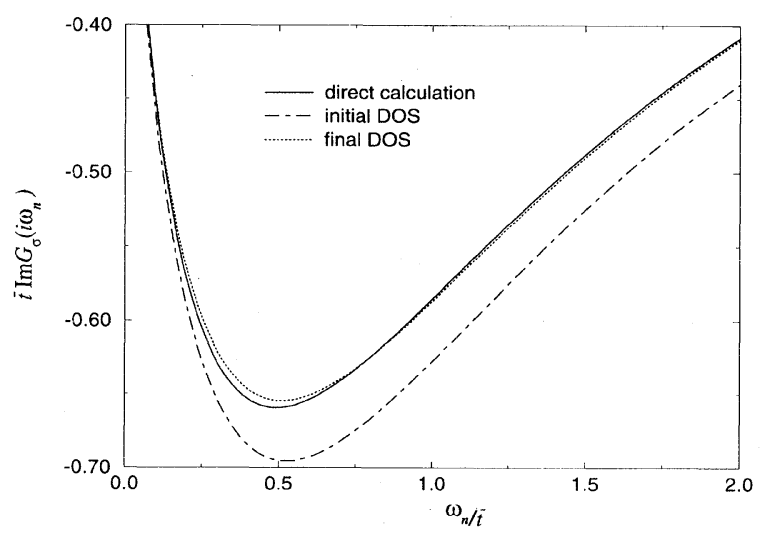

FIG. 3. Comparison between values of $G_{i i \sigma}\left(i \omega_{n}\right)$ directly obtained (continuous) and calculated from Eq. (12) with the DOS one has before (dash-dotted) and after (dotted) utilization of the maximum entropy method. 
In Fig. 3 we show typical data for the left-hand side of Eq. (12), as well as plots of the right-hand side for the initial DOS (direct substitution) and the final one, after the maximum entropy method has been applied. The residual error may still be diminished by improvements in the algorithm. Thus, the maximum entropy method allows us to obtain the correct DOS from our calculated Matsubara Green's functions.

In Fig. 4 we present, for comparison, the DOS in SC1LA when one utilizes either $\tilde{H}_{\sigma}\left(i \omega_{n}\right)$ or $H_{\sigma}\left(i \omega_{n}\right)$. One can see that the unpleasant sharp features at the position of the atomic levels that appear in the former case are not present in the latter. Both curves have been obtained through the maximum entropy method with data for $G_{i i \sigma}\left(i \omega_{n}\right)$. We have chosen the uncorrelated DOS to be a Gaussian distribution in order to make contact with results in infinite dimensions. The energy scale is set by $\bar{t} \equiv t / 2 \sqrt{d}$, which must be kept constant in the limit $d \rightarrow \infty$.

We present in Fig. 5 the DOS obtained in the SC1LA for some values of $U$. One can see that, in contrast to the ZLA, in the SC1LA the correlation gap is not present for sufficiently small values of the Coulomb interaction. This means that we have obtained a metal-insulator transition in the half-filled Hubbard model. The metallic state, however, does not correspond to a normal Fermi liquid. We have checked this by studying the frequency behavior of the self-energy, which does not show the expected $\omega^{2}$ dependence. We can also see that the DOS does not show a central peak, which would be a necessary consequence of a local (k-independent) self-energy in a normal Fermi liquid. ${ }^{7}$ We determined numerically that the critical value of $U$ for occurrence of the metal-insulator transition is $U^{*} \sim 1.5 \bar{t}$. We will compare this with results of other approaches in the next section.

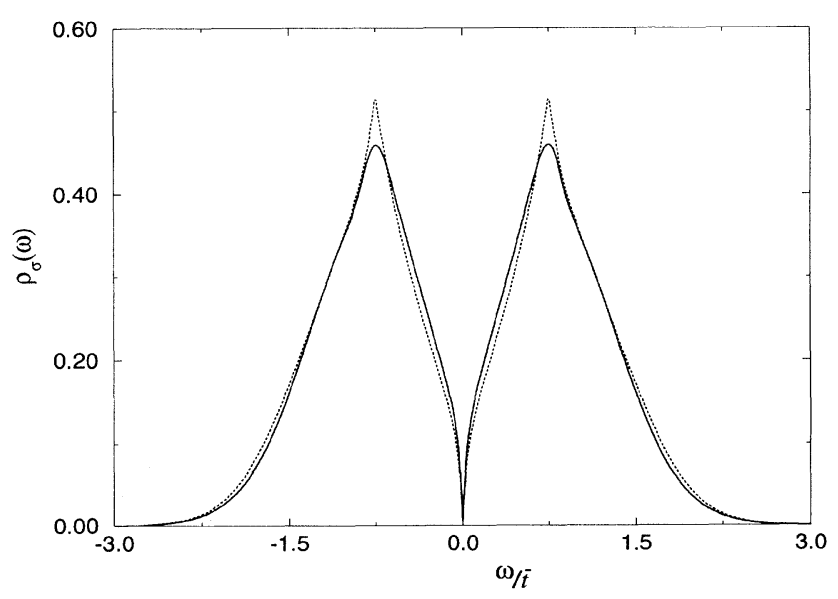

FIG. 4. Typical DOS obtained with (continuous) and without (dotted) the subtractions that lead to the substitution $\tilde{H}_{\sigma} \rightarrow H_{\sigma}$ (see text) in the renormalized one-loop diagram. Here $U / \bar{t}=1.5$.

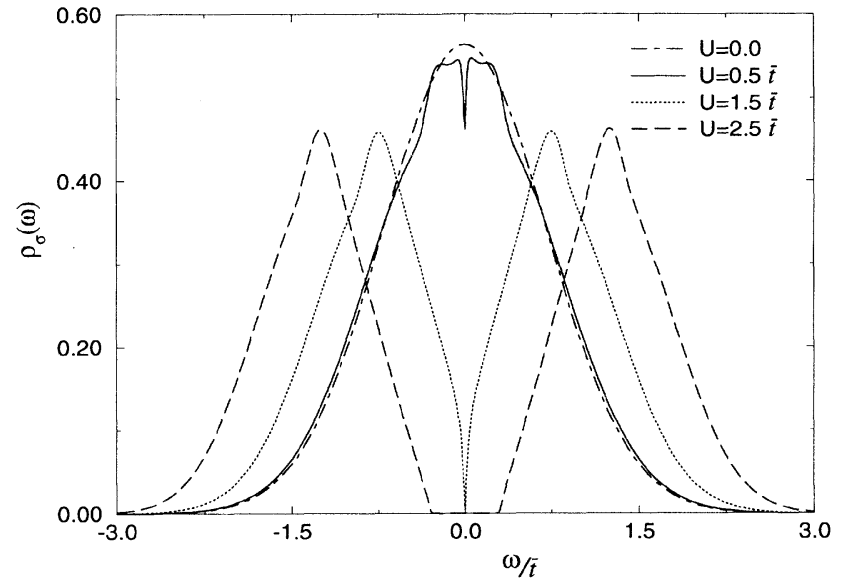

FIG. 5. DOS as a function of frequency (in units of $\bar{t}$ ) in the SC1LA for various values of the Coulomb interaction.

\section{CONCLUSIONS}

We have presented here a Green's function study of the Hubbard model, utilizing perturbation theory around the atomic limit. We have shown that it is possible to formally sum the perturbation series for the Matsubara 1PGF, writing it down in terms of an irreducible Green's function [see Eq. (6)], which can have nonlocal contributions (although these vanish in the infinite dimensional limit). The local cumulant averages appearing in the evaluation of the 1PGF (Ref. 5) can be dealt with, similarly to usual many-body theory, by means of a generalized Wick's theorem, provided that fermion operators are represented in terms of Hubbard operators. Even though this is a quite standard procedure, we have shown that terms in which the operators that define the Green's function appear in direct contraction (in the sense of Wick's theorem) are eliminated by the renormalization of the average number of particles appearing in the (zeroth-order) atomic Green's function. This renormalization is necessary in order to have a self-consistent solution for the lattice.

We have discussed common problems related to the calculation of the single-particle DOS starting from finite-temperature Green's functions. We have shown that violation of the sum rule for the frequency integral of the DOS can be due to a bad analytic continuation in the complex frequency plane. In cases where the direct substitution of $\omega+i 0^{+}$for $i \omega_{n}$ fails, a good resort is the maximum entropy method, with which one can obtain the DOS from data on the finite-temperature Green's function.

In this paper we analyzed in more detail the first correction to the zero-loop approximation (Hubbard I). By considering the one-loop diagram for the irreducible part of the 1PGF, and properly renormalizing the contribution of this single loop, we obtained a self-consistent problem whose solution for the DOS shows the existence of a metal-insulator transition for a critical value of the 
Coulomb interaction $U^{*} \sim 1.5 \bar{t}$, for a Gaussian noncorrelated DOS. Similarly to what occurs in the so-called Hubbard III approximation, ${ }^{3}$ the metallic state does not present Fermi-liquid characteristics. However, the oneloop result is much more strong coupling in nature than the Hubbard III approximation, as can be seen by the comparative behavior of the DOS in both approaches shown in Fig. 6. There, we also plot, for comparison, the DOS obtained for the Falikov-Kimball model ${ }^{14}$ in infinite dimensions, obtained from the Green's function calculated by Brandt and Mielsch. ${ }^{15}$ We can see that the agreement with this result is much better. In particular, the critical values of $U$ for the metal-insulator transition are nearly the same in both approaches.

It is worth mentioning that, although similar in structure to the Hubbard III and Falikov-Kimball equations, our results are not based on any extra assumptions as, for instance, the alloy analogy. ${ }^{3}$ They are obtained completely within perturbation theory around the atomic limit by summation of certain classes of diagrams for the Green's function. Their importance lies exactly in the fact that it is the first time a continuous crossover from the atomic limit to a noninsulating behavior in the Hubbard model was obtained within a systematic approach. The only simplifying assumption adopted here was neglecting the opposite spin contributions in the one-loop corrections. In some sense, it is equivalent to freezing one-spin species, which can probably explain the similarity with the Falikov-Kimball model. It could also be a possible reason for the absence of Fermi-liquid behavior, since we are neglecting spin fluctuations that should be important at least in the small- $U$ regime. However, due to technical difficulties in dealing with the limits $h \rightarrow 0$ and $\mu \rightarrow 0$, as commented in the previous section, we will not explore this point further here.

As a final comment, we wish to point out that the structure of the equations obtained by the perturbation method has strong similarities with current mean-field solutions of the infinite dimensional problem. ${ }^{16,8}$ The good agreement with the Falikov-Kimball model in $d \rightarrow \infty$, commented above, is also an indication that these similarities are more profound. Further investigation on this line is currently in progress.

\section{ACKNOWLEDGMENTS}

This work has been partially supported by the Brazilian agencies Conselho Nacional de Desenvolvimento $\mathrm{Ci}$ entífico e Tecnológico ( $\mathrm{CNPq}$ ), Financiadora de Estudos e Projetos (FINEP), and Fundação de Amparo à Pesquisa do Estado do Rio Grande do Sul (FAPERGS). Part of the numerical computations have been performed in the CRAY YMP-2E of the Centro Nacional de Supercomputação da Universidade Federal do Rio Grande do Sul, at Porto Alegre, Brazil.

\section{APPENDIX A}

In dealing with the average in Eq. (14) we rewrite the fermion operators in terms of Hubbard operators. ${ }^{2}$ These are defined as $X_{\alpha \beta} \equiv|\alpha\rangle\langle\beta|$, in terms of basis states belonging to the orthonormal set $\{|0\rangle,|\sigma\rangle,|\bar{\sigma}\rangle,|2\rangle\}$, which represent an empty, a singly occupied (both spin orientations), and a doubly occupied site, respectively. Hubbard operators may be fermionlike or bosonlike. The former refer to creation or annihilation of a single electron, while the latter are related to local transitions between states in which the number of electrons is either the same or differs by two. In terms of $X_{\alpha \beta}$, the relevant electron operators are $c_{\sigma}=X_{0 \sigma}+\sigma X_{\bar{\sigma} 2}, n_{\sigma}=X_{\sigma \sigma}+X_{22}$, and $n_{\uparrow} n_{\downarrow}=X_{22}$, with $\sigma= \pm 1$.

Simple commutation relations hold between these operators and the local Hamiltonian, which ensure that all the $X_{\alpha \alpha}$ are time independent, and the nondiagonal ones follow the evolution law $X_{\alpha \beta}=e^{-\varepsilon_{\alpha \beta} \tau} X_{\alpha \beta}, \varepsilon_{\alpha \beta}$ being the energy difference between the initial and final states. For instance,

$$
\begin{gathered}
\varepsilon_{0 \sigma}=\varepsilon-\mu-\sigma h, \quad \varepsilon_{\bar{\sigma} 2}=\varepsilon+U-\mu-\sigma h \\
\varepsilon_{02}=2 \varepsilon+U-2 \mu, \quad \varepsilon_{\bar{\sigma} \sigma}=-2 \sigma h
\end{gathered}
$$

A generalized Wick's theorem may be written (in compact notation) as follows:
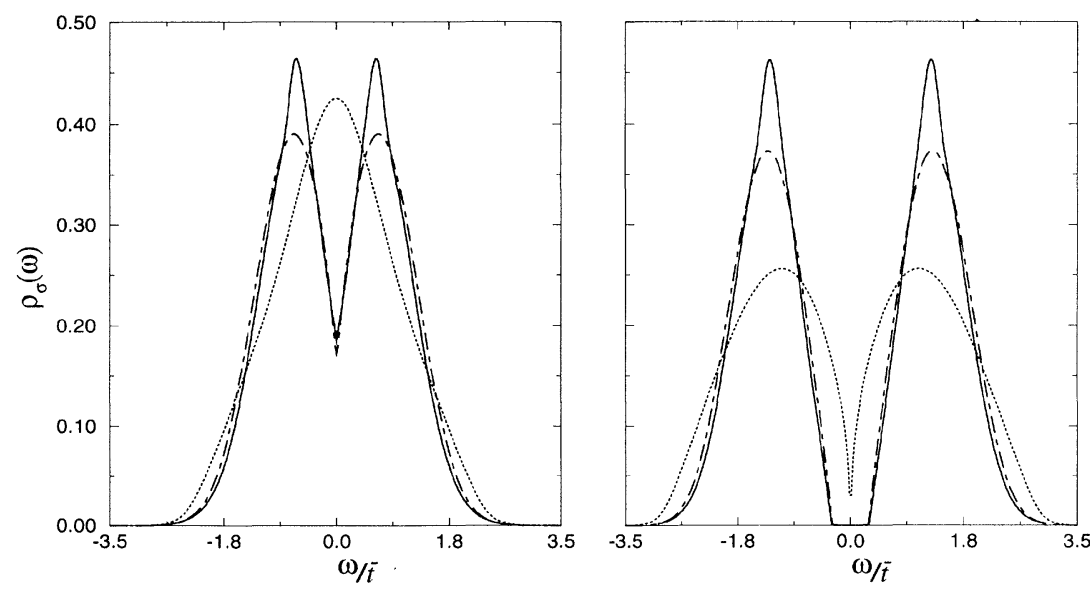

FIG. 6. Comparison between densities of states obtained by the SC1LA (continuous), Hubbard III approximation (dotted), and the Falicov-Kimball model in infinite dimensions (dashed), for $U / \bar{t}=1.25$ (left) and 2.5 (right). 


$$
\begin{aligned}
\left\langle\hat{T} X_{1} X_{2} X_{3} X_{4} \ldots\right\rangle_{0}= & -g_{1}\left(\tau_{12}\right)\left\langle\hat{T}\left\{X_{1}, X_{2}\right\}_{\tau_{2}}^{ \pm} X_{3} X_{4} \ldots\right\rangle_{0} \pm g_{1}\left(\tau_{13}\right)\left\langle\hat{T} X_{2}\left\{X_{1}, X_{3}\right\}_{\tau_{3}}^{ \pm} X_{4} \ldots\right\rangle_{0} \\
& -g_{1}\left(\tau_{14}\right)\left\langle\hat{T} X_{2} X_{3}\left\{X_{1}, X_{4}\right\}_{\tau_{4}}^{ \pm} \ldots\right\rangle_{0} \pm \cdots,
\end{aligned}
$$

where $\tau_{i j} \equiv \tau_{i}-\tau_{j},\left\{X_{i}, X_{j}\right\}^{ \pm} \equiv X_{i} X_{j} \pm X_{j} X_{i}$, and

$$
g_{1}(\tau) \equiv-\frac{e^{\gamma_{1} \tau}}{1 \pm e^{\beta \gamma_{1}}}\left[\theta(\tau) \mp e^{\beta \gamma_{1}} \theta(-\tau)\right]
$$

with $\gamma_{1}$ defined through the commutation relation

$$
\left[H_{0}, X_{1}\right]=\gamma_{1} X_{1} .
$$

In Eqs. (A2) and (A3), whenever two signs appear together the upper (lower) applies when $X_{1}$ is fermionlike (bosonlike). The subscripts on (anti)commutators indicate the time at which they are evaluated. The time dependence of the remaining operators is left implicit. Equation (A2) must be applied recursively on all the averages that are generated at each step.

In the calculation of 1PGF's, the initial averages involve only fermionlike operators, for which the nonzero anticommutators entering the contractions (with the con- vention that all contractions begin with an annihilation operator) are

$$
\begin{gathered}
\left\{X_{0 \sigma}, X_{\sigma 0}\right\}=1-n_{\bar{\sigma}}, \quad\left\{X_{\bar{\sigma} 2}, X_{2 \bar{\sigma}}\right\}=n_{\bar{\sigma}}, \\
\left\{X_{0 \sigma}, X_{\bar{\sigma} 0}\right\}=X_{\bar{\sigma} \sigma}, \quad\left\{X_{\bar{\sigma} 2}, X_{2 \sigma}\right\}=X_{\bar{\sigma} \sigma} \\
\left\{X_{0 \sigma}, X_{\sigma 2}\right\}=X_{02} .
\end{gathered}
$$

Here we have chosen to write the diagonal Hubbard operators in terms of the electron number operators. Notice that, in contrast to the expansion around the freeelectron limit, there exist nonzero contractions involving two annihilation operators, provided they refer to opposite spin states. These contractions will bring into play the bosonlike Hubbard operators. The next rule is that whenever an average contains a bosonlike operator the contractions must begin with it. The nonzero commutators involving at least one bosonlike operator are

$$
\begin{aligned}
& {\left[X_{02}, X_{20}\right]=1-n_{\sigma}-n_{\bar{\sigma}}, \quad\left[X_{\sigma \bar{\sigma}}, X_{\bar{\sigma} \sigma}\right]=n_{\sigma}-n_{\bar{\sigma}}} \\
& {\left[X_{02}, X_{\sigma 0}\right]=-X_{\sigma 2}, \quad\left[X_{02}, X_{2 \sigma}\right]=X_{0 \sigma}, \quad\left[X_{\bar{\sigma} \sigma}, X_{\sigma 0}\right]=X_{\bar{\sigma} 0}} \\
& {\left[X_{\bar{\sigma} \sigma}, X_{0 \bar{\sigma}}\right]=-X_{0 \sigma}, \quad\left[X_{\bar{\sigma} \sigma}, X_{\sigma 2}\right]=X_{\bar{\sigma} 2}, \quad\left[X_{\bar{\sigma} \sigma}, X_{2 \sigma}\right]=-X_{2 \sigma}}
\end{aligned}
$$

By the conventions stated above, the only operators that can have the role of $X_{1}$ in Eq. (A2) are $X_{0 \sigma}, X_{\bar{\sigma} 2}$, $X_{02}$, and $X_{\bar{\sigma} \sigma}$, for which the corresponding $g_{1}$ 's, after Fourier transforming in $\tau$, are
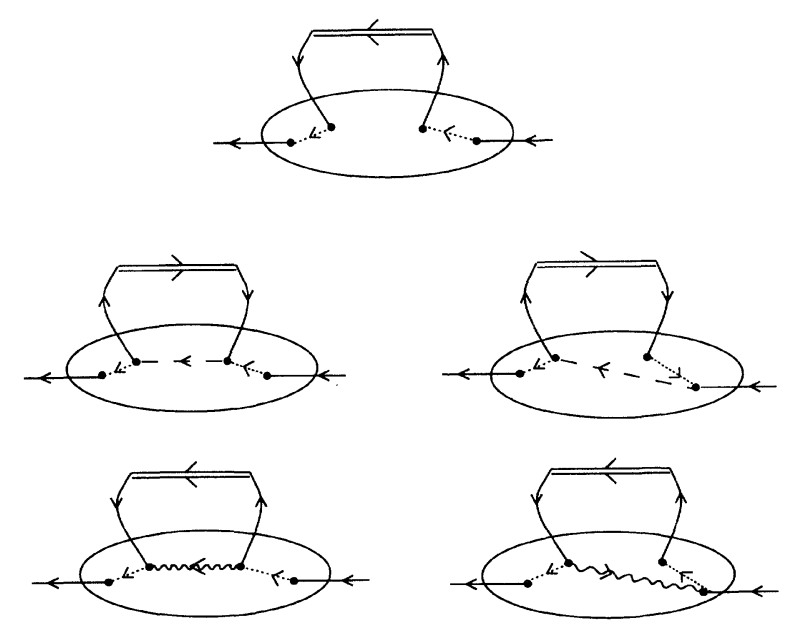

FIG. 7. Detailed view of the possible internal contractions in the four-operator average appearing in Fig. 2. Dotted lines represent either of the fermionic Green's functions of Hubbard operators, $g_{0 \sigma}$ and $g_{\bar{\sigma} 2}$, while dashed and wavy lines represent the bosonic ones, $g_{02}$ and $g_{\bar{\sigma} \sigma}$, respectively [see Eq. (A7)].

$$
\begin{aligned}
g_{0 \sigma}\left(i \omega_{n}\right) & =\frac{1}{i \omega_{n}-\varepsilon_{0 \sigma}}, \quad g_{\bar{\sigma} 2}\left(i \omega_{n}\right)=\frac{1}{i \omega_{n}-\varepsilon_{\bar{\sigma} 2}}, \\
g_{02}\left(i \nu_{n}\right) & =\frac{1}{i \nu_{n}-\varepsilon_{02}}, \quad g_{\bar{\sigma} \sigma}\left(i \nu_{n}\right)=\frac{1}{i \nu_{n}-\varepsilon_{\bar{\sigma} \sigma}},
\end{aligned}
$$

respectively, were $\nu_{n}$ is a bosonic Matsubara frequency.

Next we will show that the sum of all terms that involve a direct contraction between the external operators (those that define the Green's function) give the zerothorder local Green's function corrected for the full renormalization of the average number of particles. To do this we start with the zeroth-order form of Eq. (3) for the site-diagonal Green's function,

$$
\begin{aligned}
G_{i i \sigma}^{0}(\tau) & =-\left\langle\hat{T} X_{0 \sigma}(\tau) X_{\sigma 0}(0)\right\rangle_{0}-\left\langle\hat{T} X_{\bar{\sigma} 2}(\tau) X_{2 \bar{\sigma}}(0)\right\rangle_{0} \\
& =g_{0 \sigma}(\tau)\left\langle 1-n_{\bar{\sigma}}\right\rangle_{0}+g_{\bar{\sigma} 2}(\tau)\left\langle n_{\bar{\sigma}}\right\rangle_{0}
\end{aligned}
$$

When we take into account the perturbation through $S(\beta)$ in Eq. (3), but do not consider any other contractions, we obtain the $1 \mathrm{PGF}$ in the atomic limit,

$$
\begin{aligned}
\mathcal{G}_{\sigma}^{0}(\tau) & =g_{0 \sigma}(\tau) \frac{\left\langle\left(1-n_{\bar{\sigma}}\right) S(\beta)\right\rangle_{0}}{\langle S(\beta)\rangle_{0}}+g_{\bar{\sigma} 2}(\tau) \frac{\left\langle n_{\bar{\sigma}} S(\beta)\right\rangle_{0}}{\langle S(\beta)\rangle_{0}} \\
& =g_{0 \sigma}(\tau)\left\langle 1-n_{\bar{\sigma}}\right\rangle+g_{\bar{\sigma} 2}(\tau)\left\langle n_{\bar{\sigma}}\right\rangle,
\end{aligned}
$$

whose Fourier transform is given by Eq. (13). Notice that $\mathcal{G}_{\sigma}^{0}(\tau)$ gives the contribution of the first diagram 
(single dot) for the irreducible Green's function $\mathcal{G}_{\sigma}(\tau)$ (see Fig. 1). Hence, when evaluating other contributions to $\mathcal{G}_{\sigma}(\tau)$ we must leave out any terms coming from a direct contraction of the external operators.

Utilizing the generalized Wick's theorem, with the rules we commented above, the relevant terms originated from the diagram in Fig. 2 may be represented by diagrams with the topology shown in Fig. 7, in which we have blown up the four-legged vertex, and included internal lines to indicate the local contractions. Only diagrams like the first one survive when all spin indices in the four-operator average are the same. The remaining diagrams involve opposite spin indices between external and internal operators, in which case there is no decoupling of the four-operator average, the cumulant being equal to the simple average. Each disconnected part inside a bubble, besides the product of $g_{\alpha \beta}$ 's associated to it, includes a factor $n_{\sigma}$ or $1-n_{\sigma}$, depending on the op- erators involved in the last contraction of that particular chain. Only factors of these types appear in the final cumulant average corresponding to each bubble.

After all terms have been added we finally obtain

$$
\begin{aligned}
\Gamma_{\sigma \sigma}^{(4)}\left(i \omega_{n}, i \omega_{l}\right)= & \delta_{\omega_{l}, \omega_{n}}\left\langle n_{\bar{\sigma}}\right\rangle\left\langle 1-n_{\bar{\sigma}}\right\rangle \\
& \times\left[g_{\bar{\sigma} 2}\left(i \omega_{n}\right)-g_{0 \sigma}\left(i \omega_{n}\right)\right]^{2}
\end{aligned}
$$

and

$$
\begin{aligned}
\Gamma_{\sigma \bar{\sigma}}^{(4)}\left(i \omega_{n}, i \omega_{l}\right)= & {\left[g_{\bar{\sigma} 2}\left(i \omega_{n}\right)-g_{0 \sigma}\left(i \omega_{n}\right)\right] } \\
& \times\left\{\left[\mathcal{G}_{\sigma}^{0}\left(i \omega_{n}\right)+\mathcal{G}_{\bar{\sigma}}^{0}\left(i \omega_{l}\right)\right] g_{02}\left(i \omega_{n}+i \omega_{l}\right)\right. \\
& \left.+\left[\mathcal{G}_{\sigma}^{0}\left(i \omega_{n}\right)-\mathcal{G}_{\bar{\sigma}}^{0}\left(i \omega_{l}\right)\right] g_{\bar{\sigma} \sigma}\left(i \omega_{n}-i \omega_{l}\right)\right\}
\end{aligned}
$$

${ }^{1}$ J. Hubbard, Proc. R. Soc. London Ser. A 276, 238 (1963).

${ }^{2}$ J. Hubbard, Proc. R. Soc. London Ser. A 277, 237 (1964).

3 J. Hubbard, Proc. R. Soc. London Ser. A 281, 401 (1964).

${ }^{4}$ J. Hubbard, Proc. R. Soc. London Ser. A 285, 542 (1965); 296, 82 (1967); 296, 100 (1967).

${ }^{5}$ W. Metzner, Phys. Rev. B 43, 8549 (1991).

${ }^{6}$ M. Bartkowiak and K. A. Chao, Phys. Rev. B 47, 1616 (1993).

7 A. Khurana, Phys. Rev. B 40, 4316 (1989).

${ }^{8}$ A. Georges and G. Kotliar, Phys. Rev. B 45, 6479 (1992); M. Jarrel and T. Pruscke, Z. Phys. B 90, 187 (1993); X. Y. Zhang, M. J. Rozenberg, and G. Kotliar, Phys. Rev. Lett. 70, 1666 (1993).

${ }^{9}$ A. A. Abrikosov, L. P. Gorkov, and I. E. Dzyaloshinski, Methods of Quantum Field Theory in Statistical Physics (Prentice-Hall, Englewood Cliffs, NJ, 1963).

${ }^{10}$ N. E. Bickers, D. J. Scalapino, and S. R. White, Phys. Rev.
Lett 62, 961 (1989); F. B. Anders, N. Grewe, and A. Lorek, Z. Phys. B 83, 75 (1991).

${ }^{11}$ J. E. Shore and R. W. Johnson, IEEE Trans. Inf. Theory 26, 26 (1980); J. Skilling and R. K. Bryan, Mon. Not. R. Astron. Soc. 211, 111 (1984).

${ }^{12}$ See, for example, S. R. White, D. J. Scalapino, R. L. Sugar, and N. E. Bickers, Phys. Rev. Lett. 63, 1523 (1989); J. E. Gubernatis, M. Jarrell, R. N. Silver, and D. S. Sivia, Phys. Rev. B 44, 6011 (1991).

${ }^{13}$ There have been many works dealing with some kind of generalized Wick's theorem. See, for example, Ref. 6.

${ }^{14}$ L. M. Falicov and J. C. Kimball, Phys. Rev. Lett. 22, 997 (1969).

${ }^{15}$ U. Brandt and C. Mielsch, Z. Phys. B 75, 365 (1989).

${ }^{16}$ E. Müller-Hartmann, Z. Phys. B 74, 507 (1989); W. Metzner and D. Vollhardt, Phys. Rev. Lett. 62, 324 (1989). 